\title{
Investigation of the Airflow inside Realistic and Semi-Realistic Replicas of Human Airways
}

\author{
Frantisek Lizal $^{1, a}$, Jan Jedelsky ${ }^{1}$, Miloslav Belka ${ }^{1}$, Matous Zaremba ${ }^{1}$, Milan Maly ${ }^{1}$ and Miroslav Jicha ${ }^{1}$ \\ ${ }^{I}$ Brno University of Technology, Faculty of Mechanical Engineering, Energy Institute, Technicka 2896/2 Brno 616 69, \\ Czech Republic
}

\begin{abstract}
Measurement of velocity in human lungs during breathing cycle is a challenging task for researchers, since the measuring location is accessible only with significant difficulties. A special measuring rig consisting of optically transparent replica of human lungs, breathing simulator, particle generator and Laser-Doppler anemometer was developed and used for investigation of the velocity in specific locations of lungs during simulated breathing cycle. Experiments were performed on two different replicas of human lungs in corresponding measuring points to facilitate the analysis of the influence of the geometry and its simplification on the flow. The analysis of velocity course and turbulence intensity revealed that special attention should be devoted to the modelling of vocal cords position during breathing, as the position of laryngeal jet created by vocal cords significantly influences velocity profiles in trachea. The shapes of velocity courses during expiration proved to be consistent for both replicas; however magnitudes of peak expiratory velocity differ between the corresponding measuring points in both the replicas.
\end{abstract}

\section{Introduction}

Understanding the airflow in human lungs is important for multiple reasons. The first is to be able to prevent or reduce the harmful effects of inhaled particles; the second is to facilitate more effective, less demanding and cheap treatment of various diseases. However, measurement of air velocity in human lungs during breathing cycle is a challenging task for researchers, since the measuring location is accessible only with significant difficulties.

As in-vivo measurements are difficult to accomplish and also intrude the flow, the measurements are commonly performed in physical replicas with various level of simplification of the airways. Chang et al. [1], Isabey and Chang [2], and Menon et al. [3] used Hot Wire Anemometry in a 3:1 scaled replica with four generations of branching. Several other researchers used Particle Image Velocimetry (PIV) and optically transparent replicas [4,5]. Ramuzat and Riethmuller [5] used substantially idealised replica with smooth cylindrical tubes, whereas Adler et al. [6] used a replica based on Horsfield's [7] and Weibel's [8] models of lungs. Only few studies have been performed using Laser-Doppler Velocimetry (LDV). Corieri and Riethmuller [9] measured velocity in a glass single bifurcation. Similar simple replica was used also by Peattie and Schwarz [10]. On the contrary Corcoran and Chigier [11] used more realistic geometry originating from a cadaver lung cast.
It is apparent from the preceding list that the flow was studied in variety of replicas with different level of simplification. Question may therefore arise whether the results are comparable and how important is to have a precise replica of lung geometry. Lin et al. [12] performed numerical simulation focused on upper part of airways. They compared two airway geometries (with and without the oral cavity and larynx) and demonstrated that especially the glottal area must be included in the replica, because it substantially shapes the laryngeal jet. The need for realistic geometry with asymmetric branching was also emphasized by Grosse et al. [13].

Despite the intelligible demands to produce the most realistic replicas of the airways, the motivation to simplify the geometry remains strong. The optical measuring methods suffer from optical distortions of the laser sheet or beam. The distortions can be minimized using cylindrical tubes instead of realistic curved shapes of airway branches. Another simplification is related to the inclusion of the oral cavity. The flow below the larynx should be influence mainly by the strong constriction of the flow in vocal cords. However, the transition of small velocity fluctuations induced in the oral cavity down to the trachea remains questionable.

To gain more data on the topic of the reasonable simplification of the lung geometry, we developed two replicas of human airways and performed LDV measurements during simulated breathing cycle. Results

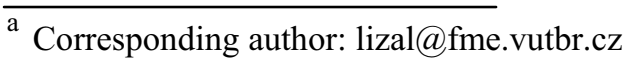


acquired in two cross-sections in trachea are presented in this paper.

\section{Methods}

Measurement was performed using two airways replicas that originate from the same dataset. Breathing mechanism simulated sinusoidal course of breath. Two different laser-Doppler-based methods were used for velocity measurements. In this text we use the term "model" for the digital or virtual representation of the airways and the term "replica" for the physical replica of airways used for experiments.

\subsection{Human airways replicas}

The minimization of the laser beam distortions can be achieved by matching the refractive index of the replica wall with the refractive index of the flowing liquid. This method is frequently used for investigations using PIV (e.g. [13]). We used alternative approach which allows us to use real aerosols, i.e. the mixture of particles and air. Both the replicas have thin walls, therefore the beam trajectory changes only subtly during the transition of the beam through the wall.

Digital reference model of human lungs published by Schmidt et al. [14] was processed and served as a base of both the replicas. As the Schmidt's model does not include airways above trachea, it was necessary to complement the model with upper airways geometry. Based on the results of Lin et al. [12], who demonstrated the main role of glottal area, the first replica (from now on called realistic) was built without the oral cavity. The geometrical data of upper airways were acquired in collaboration with St. Anne's University hospital in Brno. The replica itself was produced by brush coating of several layers of silicone on a soluble core, which was produced by rapid prototyping method. The first replica is called realistic (RR) throughout this text.

The original airway geometry was then simplified to produce the second replica (from now on called semirealistic, SR). It has smooth cylindrical walls made of glass and bifurcation pieces directly produced by rapid prototyping. The volumes and lengths of the branches were preserved identical with the RR, as were the angles of branchings. We decided to complement the SR with the oral cavity, so it could be used for other experiments focused on the measurement of inhaled aerosols deposition. The details on development of the replica were published in Lizal et al. [15].

Digital geometries of both the models are available for numerical simulations.

\subsection{Experimental setup}

Experiments were performed with the RR at first. The measuring rig consisted of in-house developed breathing simulator which generated sinusoidal course of flow rate with tidal volume 1.0 liter and duration of one breath $4 \mathrm{~s}$. Tracing particles in the size of $3 \mu \mathrm{m}$ were generated from di-2-ethylhexyl sebacate by Condensation monodisperse aerosol generator (TSI 3475) and led to the replica for both phases of the breathing cycle, inspiration and expiration. Measurements were performed in multiple cross-sections of the replicas, however only two crosssections in trachea are presented in this paper due to space limitations. Cross-section T-a lies $30 \mathrm{~mm}$ above the cross-section $\mathrm{T}-\mathrm{b}$, which is right above the first bifurcation.

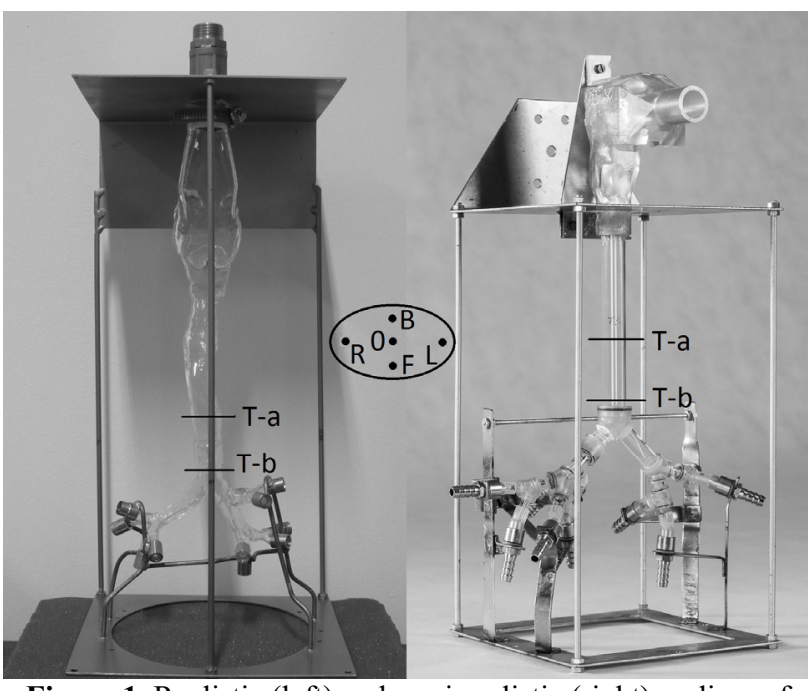

Figure 1. Realistic (left) and semi-realistic (right) replicas of human airways with measuring cross-sections T-a and T-b.

The measuring points are designated as follows: $\mathrm{F}$ points in the front of the cross-section, $\mathrm{B}$ - points in the back of the cross-section, $\mathrm{R}$ - points on the right and $\mathrm{L}-$ points on the left in the cross-section. Point in the middle is designated as 0 . Orientation of directions corresponds to the body directions (right is always on the side of right hand, etc.). Two measuring points were set in each direction. The point closer to the middle (in approximately $30 \%$ of a section radius) is designated as 1 . The point closer to the wall (in approximately $62 \%$ of a section radius) is designated as 2 .

The whole label of measuring point is then e.g. T-aB2, which means: Trachea, cross-section T-a, point in the back side of the cross-section, closer to the wall.

Data files containing arrival time, transit time and velocity of each particle were processed in MATLAB R2007b software. Temporally fluctuating axial velocities $\mathrm{v}^{*}$ were calculated in $30 \mathrm{~ms}$ time bins $( \pm 15 \mathrm{~ms}$ around each particle). The duration of each time bin was determined to be so short that the expected change in the mean value of velocity during the inspected time bin was much smaller than the predicted fluctuating velocity. Normalized fluctuating axial velocity (axial turbulence intensity, TI) was calculated as TI $=\mathrm{v}^{*} / \mathrm{v}_{\mathrm{m}}$; where the average velocity in the cross section $\mathrm{v}_{\mathrm{m}}$ was calculated from the measured actual flow rate. Only time bins with more than 9 particles were processed. 


\subsection{Velocity measuring devices}

\subsubsection{Measurements in the realistic replica}

Phase-Doppler anemometry (PDA) was used for 1-component measurement of velocity, size and arrival time of individual air-borne aerosol particles flowing through the realistic airway replica (Jedelsky, J., Lizal, F. and Jicha, J., Time-resolved characteristics of oscillatory particle-laden air flow in a realistic human airway model, Experiments in Fluids, under review, 2014).

The classical 1-component PDA system (Dantec Dynamics A/S, Skovlunde, Denmark) was set for measurement of the first-order refracted light from particles at forward scattering angle $50-65^{\circ}$. The PDA was chosen instead of the simpler LDV, (1) to verify particle size and also (2) to reduce unwanted sources of noise in velocity data such as reflections and multipleparticle scattering.

\subsubsection{Measurements in the semi-realistic replica}

Two-component LDV system Dantec Dynamics Flow explorer 9065x0341 (Dantec Dynamics A/S, Skovlunde, Denmark) was employed for time-resolved point-wise measurement of velocity of individual particles flowing inside the SR. Only one velocity component, corresponding to the local axial flow direction, was recorded. A built in, diode-pumped solid-state, laser generated a beam with $660 \mathrm{~nm}$ wave length. The beam was split into two parallel beams where the frequency of one of them was shifted by $80 \mathrm{MHz}$. A converging transmitting/receiving lens with $300 \mathrm{~mm}$ focal length was used to form an ellipsoidal probe volume with size app. $0.1 \times 0.1 \times 1 \mathrm{~mm}$ at the beam crossing point. Both the shifted and un-shifted beams featured light power about $30 \mathrm{~mW}$ at the measurement position. The LDV worked in the back-scatter mode and used the Dantec BSA P80 flow and particle processor for signal processing. That arrangement provides the velocity results with uncertainties larger than those in the PDA setup.

BSA Flow software v5 was used for the measurement control, data recording and processing with these general settings: photomultiplier sensitivity 700-1000 mW, signal gain 16-22 dB, record length: auto-adaptive with minimum and maximum record length set to 32 and 256 respectively, burst detector SNR level 1-4 dB. Particular settings of these values depended on the signal strength and position of the measured point. The cyclic flows were recorded always for two full cycles with velocity span 6$38 \mathrm{~m} / \mathrm{s}$ and velocity center at $0 \mathrm{~m} / \mathrm{s}$ (for all measurements). A minimum of 10,000 samples was acquired at each measured point.

\section{Results and discussion}

Velocity courses for RR and SR are compared in Fig.2. Velocities are positive during inspiration and negative during expiration. Each data point represents one particle which arrived into the measuring volume of PDA or LDV. Data from points R2, T-b-R1 and T-b-L2 had to be removed from the comparison due to problems with LDV data validation during measurement caused by complicated optical access.

Data were interpolated using cubic-B spline, which splits the input data into pieces and fits each segment with discrete Bezier splines. The dashed line represents the RR, the full line represents SR. Graphs in the left column show velocities in the cross-section $\mathrm{T}-\mathrm{a}$; graphs in the right column show velocities in the cross-section T-b. Flow development in the trachea can therefore be seen by comparing the left and right graph.

Comparison of velocity courses in the middle of the trachea (T-a-0 and $\mathrm{T}-\mathrm{b}-0)$ shows that the profiles are almost sinusoidal with minimal distortions. Magnitudes of peak inspiration and expiration are identical for both cross-sections in the SR, however significant increase of peak velocities can be seen for RR. It should be noted, that the areas of T-a and T-b cross-sections are almost identical, therefore the increase of velocity during inspiration is not caused by an airway constriction, but rather by the flow development, namely by the laryngeal jet shift and decay. It is worth noting that the replicas have different shapes of glottal geometry. It is obvious that the vocal chords strongly influence the downstream flow and therefore careful attention should be paid to their simulation.

The flow during expiration is governed by mixing of flow from the two daughter tubes. RR and SR differs mainly in the curvature of the left branch (the longer main bronchus), which is straight in the SR, but whose axis curves up and back in the RR.

Interesting velocity course, which is reproduced in both replicas, can be seen in both points in the back of the cross-section T-b during expiration. There are two distinct decelerations (compared to an ideal sinus curve) in time instants about $2.5 \mathrm{~s}$ and $3.5 \mathrm{~s}$. It means that in those time instants either a vortex with the opposite velocity crossed the measuring volume, or the jet created by mixing of the two daughter tubes shifted due to e.g. detachment of the flow from the main bronchus wall. Indication of the same phenomenon can be observed even in the T-a-B points as a small change of the slope on the velocity curve in time instants around $2.4 \mathrm{~s}$. The fact that this observation was made for both replicas means that the phenomenon is not influenced by the left main bronchus curvature.

Differences in magnitudes of peak inspiration velocity are almost negligible for the whole $\mathrm{T}$-a cross-section, but become noticeable in T-b. Velocities are lower in the SR especially in points $\mathrm{B} 1$ and $\mathrm{B} 2$, which means that the laryngeal jet shifted to the front and right in the SR. Expiratory flow is more uniform in the SR, which is indicated by higher velocities in points closer to the wall and lower velocities in the middle compared to RR.

Comparison of TI (Fig. 3) reveals that TI is higher during expiration than during expiration for both replicas and all points. TI in SR seems systematically higher, but this observation should be discussed carefully. As was notices in the previous chapter, LDV worked in backscatter mode and this arrangement results in larger uncertainties. 

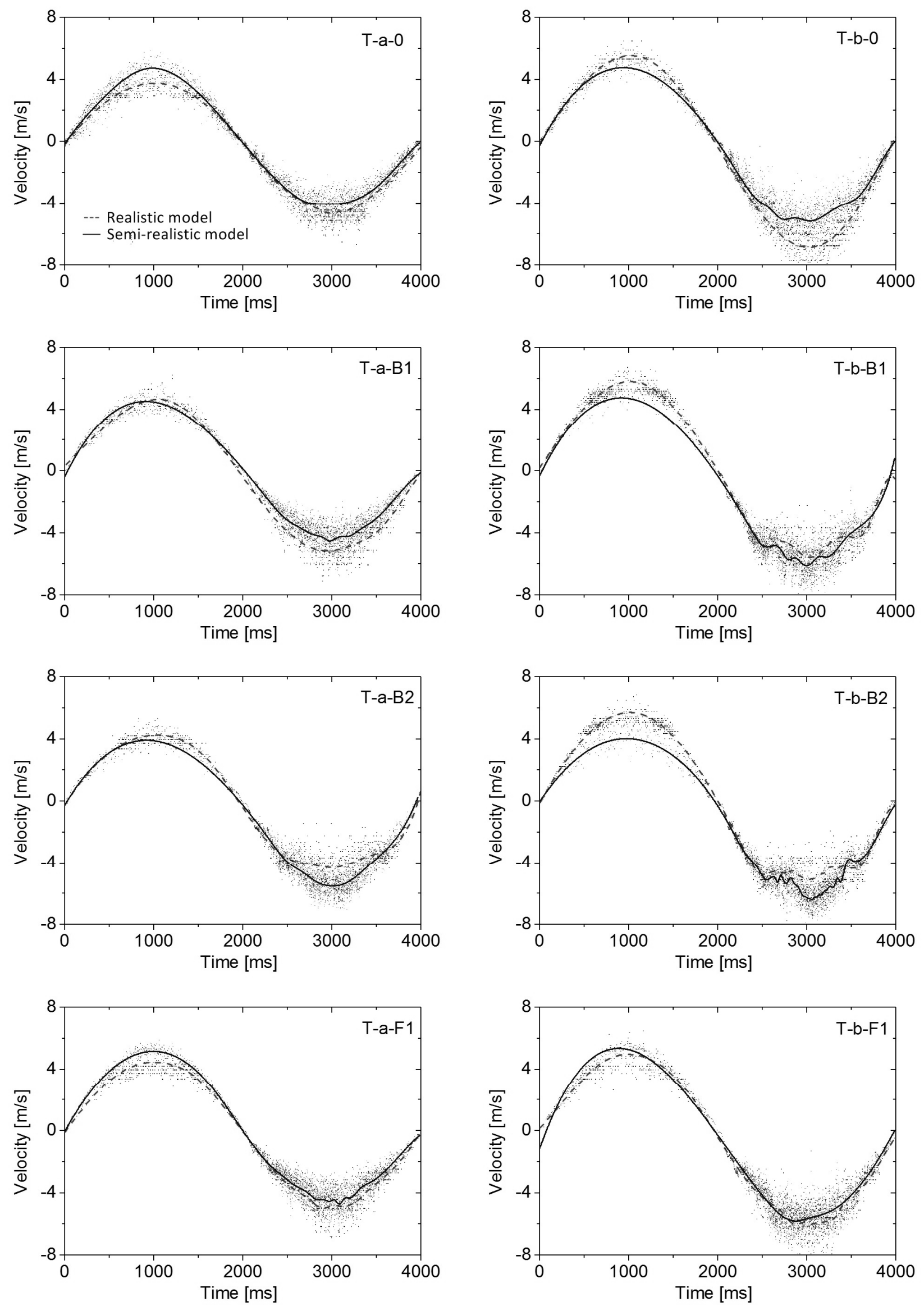

Figure 2. Velocity courses in trachea cross-sections $a$ (left column) and $b$ (right column) in corresponding points 

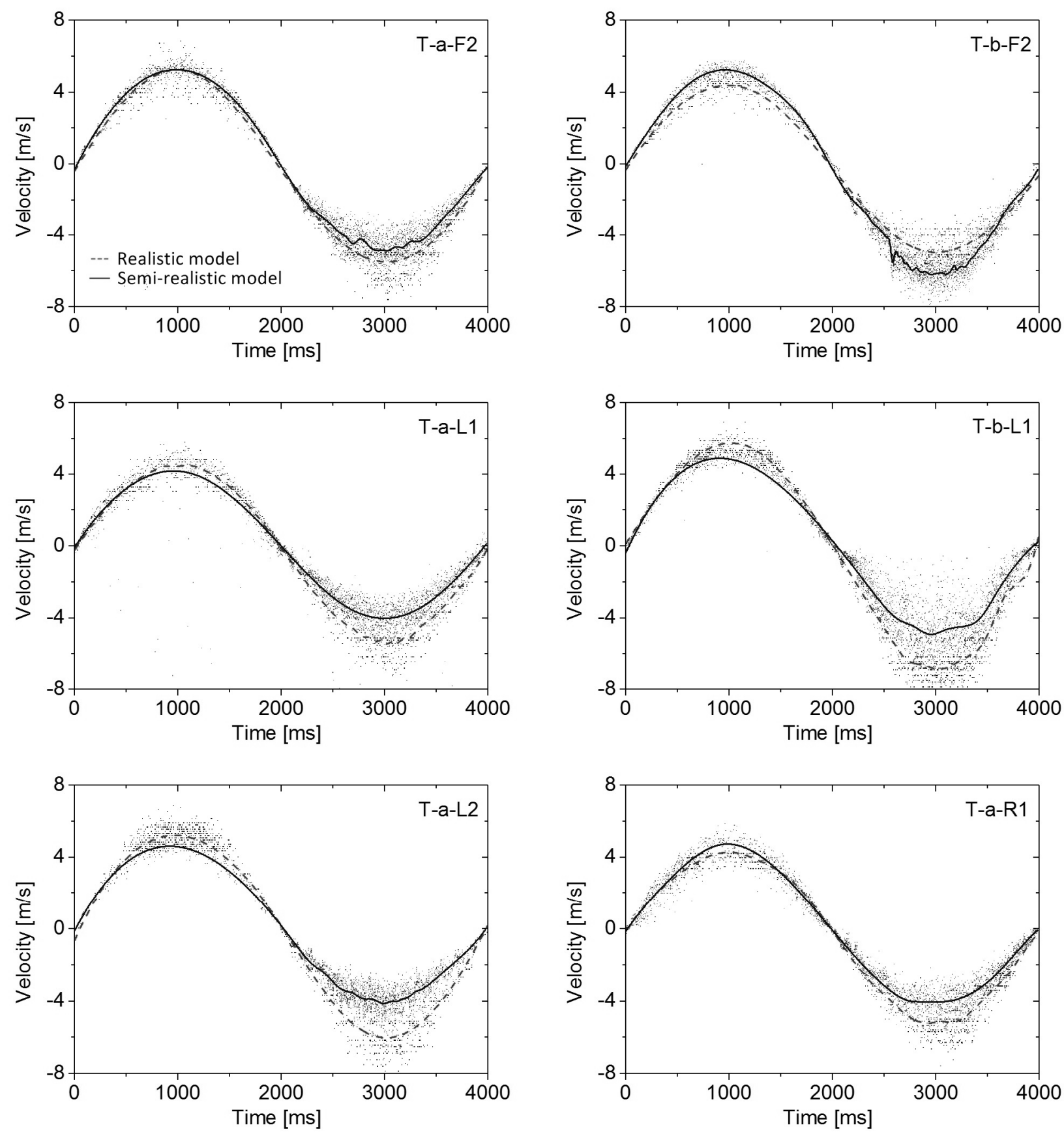

Figure 2 - continued. Velocity courses in trachea cross-sections $a$ (left column) and $b$ (right column) in corresponding points

LDV also does not allow for adjustments of transmitting optics, which also could contribute to measurement uncertainties. Therefore the variance in velocities might be artificial and real TI might be lower. However, the difference between TI for SR and RR reaches $20 \%$ in some points during expiration, which cannot entirely be attributed to uncertainties.

Apart from the uncertainties, the differences between SR and RR during inspiration are probably incurred by the presence of the oral cavity in SR, whose complex geometry induces small velocity fluctuations.

Interesting development of TI can be seen in points Ta- 0 and T-a-R1, where TI rapidly increases from the beginning of the cycle to $0.3 \mathrm{~s}$ and then gradually decreases until $1.6 \mathrm{~s}$, when a sharp decrease occurs. The only point with lower TI for SR is T-a-F2. No difference can be seen between interpolated velocities in the same point.

TI is significantly higher in SR for all points during respiration. The airways of both replicas below trachea are based on the same model geometry and the only exception is straightened tubes in SR. We would therefore expect lower TI in SR. The increase can be attributed to the connection of glass tubes with plastic bifurcations. The connection is not perfectly smooth and the flow around the sharp edge can induce fluctuations, which pronounce further in the airways.

Very high TI was observed in T-b-L1, it reaches $45 \%$. It is a result of mixing of air from the daughter branches. The flow is distributed to the daughter tubes in a ratio 1:2, where higher flow rate goes to the right branch, which is straight and has larger diameter. 

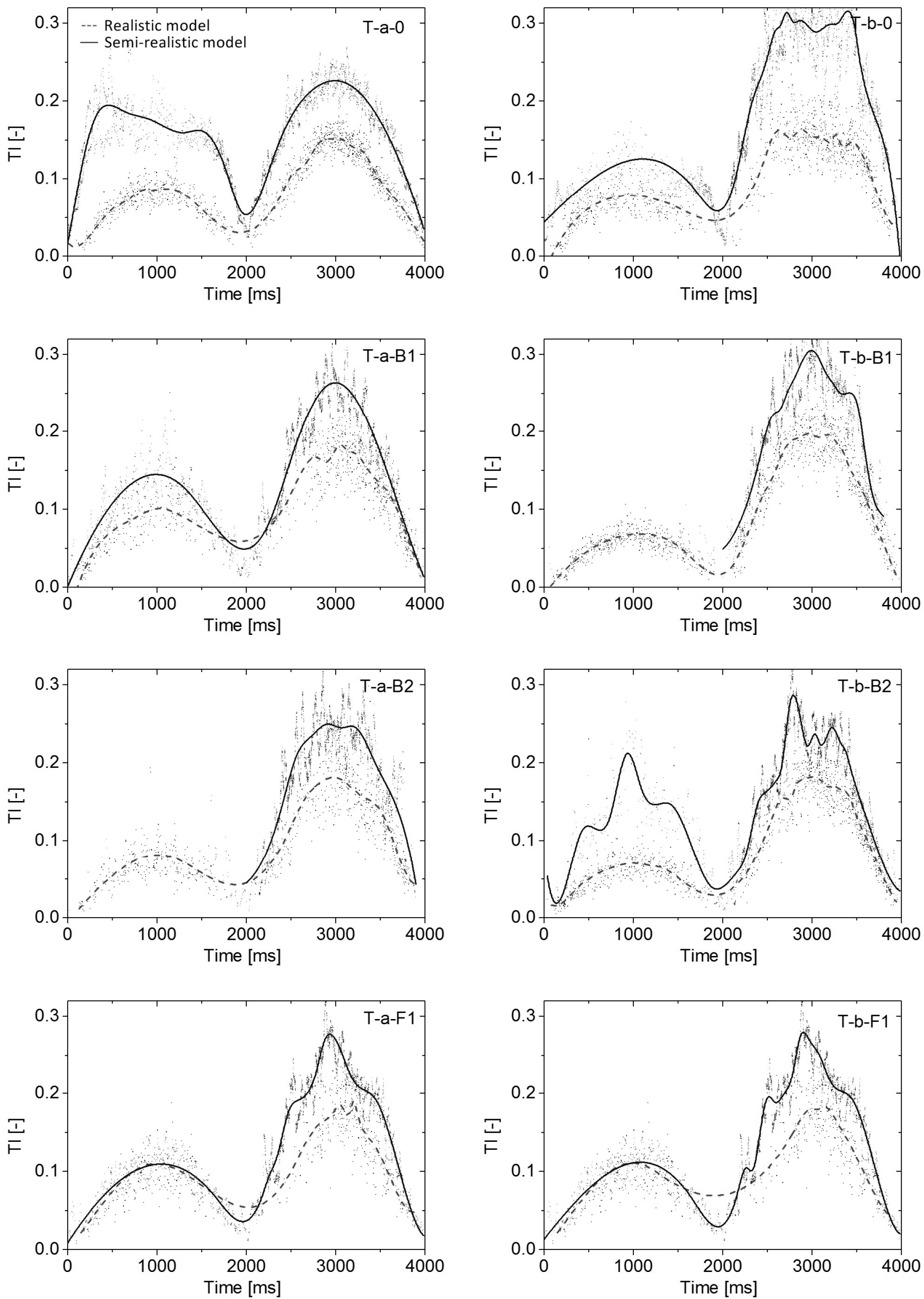

Figure 3. Turbulence intensities in trachea cross-sections $a$ (left column) and $b$ (right column) in corresponding points $\left(\mathrm{TI}=\mathrm{v}^{*} / \mathrm{v}_{\mathrm{m}}\right)$ 

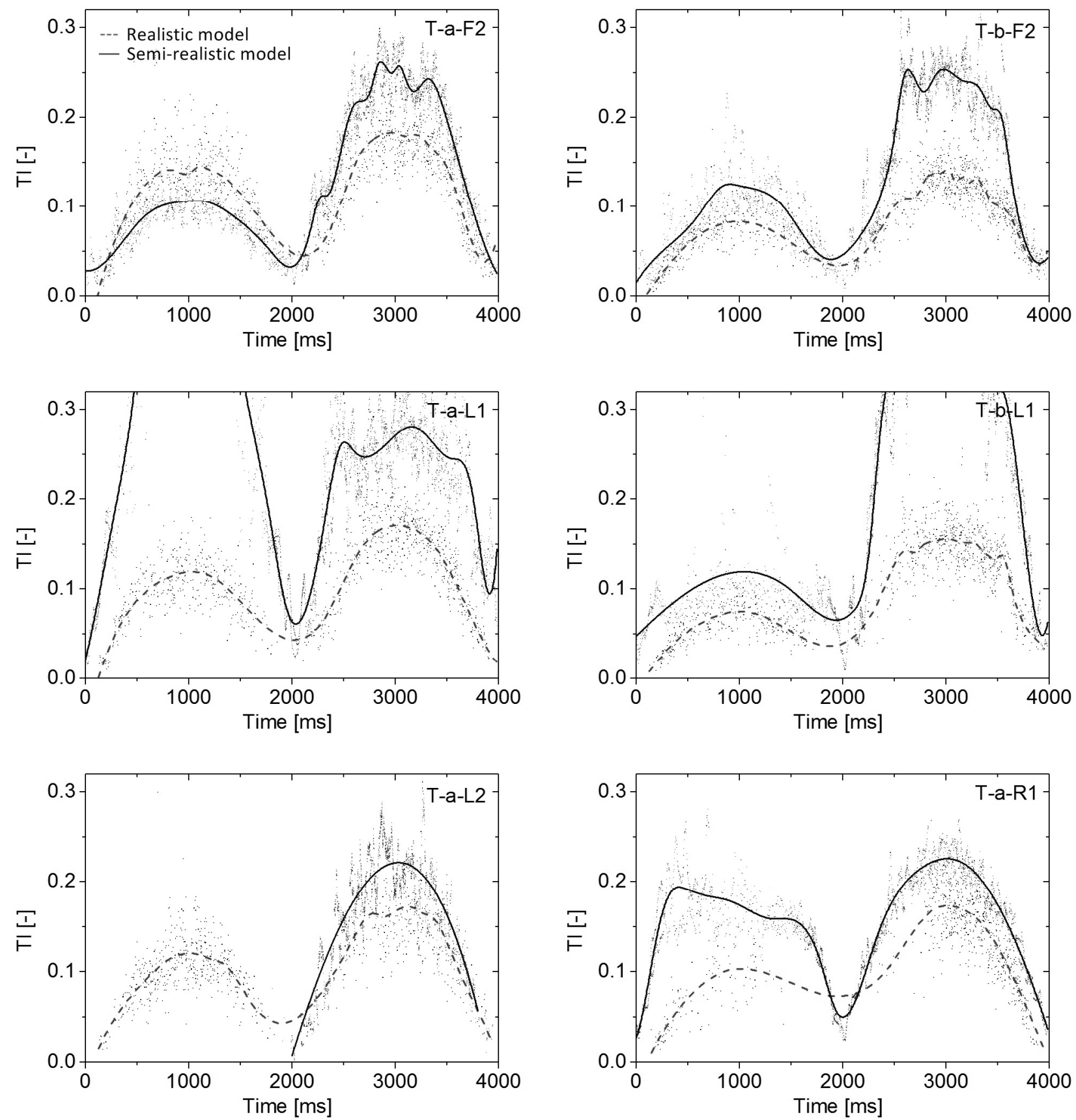

Figure 3 - continued. Turbulence intensities in trachea cross-sections $a$ (left column) and $b$ (right column) in corresponding points $\left(\mathrm{TI}=\mathrm{v}^{*} / \mathrm{v}_{\mathrm{m}}\right)$

Point L1 is located above the left main bronchus, where strong secondary velocities can be expected as a result of flow through the curved tube.

Another high peak of TI can be seen in the point T-a-L1, however LDV data from this point were of worse quality and should be taken with restraints, because the variability of velocity might be of artificial origin.

\section{Conclusions}

Comparison of velocity measurements performed on two different airway replicas revealed that the shape of vocal cords and the whole glottal area influence significantly the flow in the trachea and the first bifurcation during the inspiration phase. It is known that the laryngeal jet formed in the glottis governs the deposition of particles in the area downstream. Therefore it is difficult to compare local deposition patterns in replicas with different glottal areas. This problem is becoming more important nowadays, when numerical simulations of aerosol deposition allow for detailed comparison of high resolution deposition data, which should be experimentally validated. In that case identical geometries must be used for numerical simulation and experiment to get comparable data.

Based on the TI analysis, we can conclude that the influence of the oral cavity might result in increased 
velocity fluctuations. However this conclusion is supported by less accurate LDV measurement and should be confirmed by repeated measurement performed with PDA.

Results acquired on the two replicas during expiratory phase show generally similar characteristics. TI in trachea is always higher during expiration than during inspiration as a result of mixing of the flows from daughter branches.

Digital model geometries of both replicas are available for numerical simulations, which we believe will give deeper understanding to the flow mechanics in lungs. The laser-Doppler-based methods acquire pointwise data only, therefore the combination with numerical simulation can elucidate the origin and development of the structures, which are experimentally detected, but cannot be fully described as such. Experimental methods should on the other hand used for the necessary validation of numerical simulations.

\section{Acknowledgement}

Frantisek Lizal was supported by the project CZ.1.07/2.3.00/30.0039 of Brno University of Technology. The results published here are the outcome of collaboration within the projects P105/11/1339 supported by the Czech Science Foundation and NETME Centre, regional research and development center established and founded from Operational Programme Research and Development for Innovation in a frame of project NETME Centre (New Technologies for Mechanical Engineering), Reg. no.: CZ.1.05/2.1.00/01.0002 and supported in a sustainability phase by project NETME CENTRE PLUS (LO1202) financed by The Ministry of Education, Youth and Sports in a frame of the "National sustainability program I".

\section{References}

1 H. K. Chang and O. A. Elmasry, Respiration Physiology 49 (1), 75 (1982).

2 D. Isabey and H. K. Chang, Respiration Physiology 49 (1), 97 (1982).

3 A. S. Menon, M. E. Weber, and H. K. Chang, Respiration Physiology 55 (2), 255 (1984).

4 I. Horschler, C. Brucker, W. Schroder, and M. Meinke, European Journal of Mechanics B-Fluids 25 (4), 471 (2006); K. Adler and C. Brucker, Experiments in Fluids 43 (2-3), 411 (2007).

5 A. Ramuzat and M. L. Riethmuller, presented at the 11th Int. Symp. on Appl. of Laser Techniques to Fluid Flows, 2002 (unpublished).

$6 \quad$ K. Adler, W. Schröder, and C. Brücker, presented at the 13th Int. Symp. on Appl. Laser Techniques to Fluid Mechanics, Lisbon, Portugal, 2006 (unpublished).

7 K. Horsfield, G. Dart, D. E. Olson, G. F. Filley, and G. Cumming, J Appl Physiol 31 (2), 207 (1971).

8 E. Weibel, Morphometry of the Human Lung. (Springer Verlag and Academic Press, Berlin, New York, 1963).
9 P. Corieri, M. L. Riethmuller, and Ieee, Iciasf 89 Record: International Congress on Instrumentation in Aerospace Simulation Facilities, 226 (1989).

10 R. A. Peattie and W. Schwarz, Journal of Biomechanical Engineering-Transactions of the Asme 120 (5), 584 (1998).

11 T. E. Corcoran and N. Chigier, Journal of Aerosol Medicine-Deposition Clearance and Effects in the Lung 13 (2), 125 (2000).

12 C. L. Lin, M. H. Tawhai, G. McLennan, and E. A. Hoffman, Respiratory Physiology \& Neurobiology 157 (2-3), 295 (2007).

13 S. Grosse, W. Schroder, M. Klaas, A. Klockner, and J. Roggenkamp, Experiments in Fluids 42 (6), 955 (2007).

14 A. Schmidt, S. Zidowitz, A. Kriete, T. Denhard, S. Krass, and H. O. Peitgen, Computerized Medical Imaging and Graphics 28 (4), 203 (2004).

15 F. Lizal, J. Elcner, P. K. Hopke, J. Jedelsky, and M. Jicha, Proceedings of the Institution of Mechanical Engineers Part H-Journal of Engineering in Medicine 226 (H3), 197 (2012). 